\title{
Altered E-cadherin and epidermal growth factor receptor expressions are associated with patient survival in lung cancer: a study utilizing high-density tissue microarray and immunohistochemistry
}

\author{
George Deeb ${ }^{1,2}$, Jianmin Wang ${ }^{3}$, Nithya Ramnath ${ }^{3}$, Harry K Slocum ${ }^{1,2}$, Sam Wiseman ${ }^{4}$, \\ Amy Beck ${ }^{1,2,3,4}$ and Dongfeng Tan ${ }^{1,2,3,4}$ \\ ${ }^{1}$ Department of Pathology; ${ }^{2}$ Department of Laboratory Medicine; ${ }^{3}$ Department of Medicine and ${ }^{4}$ Department \\ of Surgery Roswell Park Cancer Institute, State University of New York, Buffalo, NY 14263, USA
}

\begin{abstract}
E-cadherin (E-cad) and epidermal growth factor receptor (EGFR) are important cell adhesion and signaling pathway mediators. This study aimed to assess their expression in lung adenocarcinoma (AdC) and squamous cell carcinoma (SCC) and their association with clinicopathologic variables. In all, 130 resectable lung cancers (stages I-IIIA) were studied using a high-density tissue microarray. Two to three cores from each case were arrayed into three blocks using a Beecher system. Immunohistochemistry was performed using an avidinbiotin complex method and monoclonal antibodies against E-cad and EGFR. Unequivocal membrane staining in $>10 \%$ of tumor cells was considered as a positive expression of E-cad and EGFR. Markers expression and coexpression were analyzed against clinicopathologic variables (age, gender, smoking status, performance status, weight loss, histology, grade, stage, and lymph node involvement) and patient survival. There were 118, 126, and 115 cases that were fully assessable for E-cad, EGFR, and both markers, respectively. For E-cad, 65 cases $(55 \%)$ were positive $(+), 53(45 \%)$ were negative $(-) ; 23$ cases of the negative group had only cytoplasmic staining. For EGRF, 43 cases $(34 \%)$ were $(+)$, and $83(66 \%)$ were $(-)$. There was no significant association between E-cad or EGFR, and any of the clinicopathologic variables except for an association between EGFR( + ) and SCC histologic type. Both negative and cytoplasmic staining of E-cad correlated with shorter patient survival with $P=0.008$ and 0.002 , respectively. EGFR expression did not correlate with patient survival; however, patients with E-cad(-)/EGFR(+) phenotype had poorer survival than those with E-cad(+)/EGFR(-) $(P=0.026)$. Our study suggests that lung AdC and SCC may be stratified based on expression of E-cad and EGFR with the E-cad(-)/EGFR(+) expression having a worse disease outcome. Moreover, the cytoplasmic expression of E-cad may represent an altered localization of this protein in association with tumorigenicity. Modern Pathology (2004) 17, 430-439, advance online publication, 23 January 2004; doi:10.1038/modpathol.3800041
\end{abstract}

Keywords: Non-small-cell lung cancer; adenocarcinoma; squamous cell carcinoma; E-cadherin; epidermal growth factor receptor; tissue microarray; immunohistochemistry

Recently, several biological markers have been recognized as prognosticators, as well as indicators of potential therapeutic targets for different types of

Correspondence: D Tan, Departments of Pathology and Cancer Genetics, Roswell Park Cancer Institute, State University of New York, Buffalo, Elm and Carlton Streets, Buffalo, NY 14263, USA. E-mail: dongfeng.tan@roswellpark.org

Part of this report has been accepted for poster presentation in American Society of Clinical Oncology (ASCO) annual meeting, Chicago, 2003.

Received 03 July 2003; revised 09 September 2003; accepted 17 October 2003; published online 23 January 2004 human cancer including non-small-cell lung cancer (NSCLC). Owing to the complexity of the molecular biology of NSCLC, multiple factors, including those involved in the cell growth and cell cycle control, morphogenesis, apoptosis, angiogenesis, and metastatic adhesion, have been studied with the aim of creating biological risk assessment and biological staging models for NSCLC. ${ }^{1,2}$ E-cadherin (E-cad) is a cell-cell adhesion transmembrane molecule that connects epithelial cells via homotypic calciumdependent interactions. E-cad has an important role in cell adhesion specificities and morphogenesis, and it may have a signaling effect through interaction 
with intracellular cytoskeleton where the tyrosine kinase of the src family is localized..$^{3,4}$ In addition, because cell discohesiveness and detachment are important for tumor invasiveness, decreased expression or loss of E-cad, quantitatively and qualitatively, may facilitate tumor invasiveness and metastasis. ${ }^{4}$ Epidermal growth factor receptor (EGFR), a member of the superfamily of receptors with intrinsic tyrosine kinase activity, ${ }^{5}$ plays a role in signal transduction inducing cell proliferation and differentiation. Furthermore, it has been suggested that these two molecules have a reciprocal relationship in controlling cell-to-cell adhesion and cell proliferation and differentiation. ${ }^{6}$ In vitro, stimulation of EGFR resulted in reduced intercellular adhesion in esophageal cancer cell lines and blockage of EGFR activity was reported to upregulate E-cad in lung cancer cell lines. ${ }^{7,8}$ Clinically, reduced E-cad expression was found to correlate with dedifferentiation, invasiveness, and poor prognosis in NSCLC, although there was an inconsistency regarding its dependability as a prognostic factor. $^{9-11}$ On the other hand, overexpression of EGFR was reported to be associated with lymph node involvement and poor prognosis in NSCLC. ${ }^{11,12}$

Few investigations addressed the coexpression of E-cad and EGFR, ${ }^{13}$ and the relation between this expression and patient survival in NSCLC. The aim of this study was to utilize a high-throughput tissue microarray (TMA) and immunohistochemistry (IHC) for assessment of E-cad and EGFR expressions in two major subtypes of NSCLC, adenocarcinoma (AdC) and squamous cell carcinoma (SCC), to evaluate their association with clinicopathologic variables and patient survival, and to address their possible value as prognosticators.

\section{Materials and methods}

\section{Patients Selection}

The study group was retrospectively selected from Roswell Park Cancer Institute patients between 1996 and 1999. The selection criteria were having lung AdC or SCC as a primary diagnosis, complete surgical resection of the tumor (macroscopically and microscopically) as the initial treatment modality, availability of adequate archival tissue for evaluation, and complete clinicopathologic data. Patients who died within 1 month of the operation were excluded from the study to avoid the bias of perioperative mortality. In all, 130 patients met the study criteria. Patients were followed through 3/6/ 2002 and the median follow-up time was 57.2 months.

Clinicopathologic data included age (years), gender (male or female), ECOG performance status ( $0 \mathrm{vs}$ $1,2$, or 3$),{ }^{14}$ weight loss (presence or absence), smoking history (never or ever), tumor histology (AdC, SCC, or large cell carcinoma), tumor grade (well, moderate, or poor differentiation), pathologic stage (I vs II and IIIA), positive lymph nodes (0 vs 1 and 2), and last clinical follow-up date or date of death from lung cancer. This study was performed under an Institutional Review Board-approved protocol to investigate molecular markers relevant to lung cancer pathogenesis.

\section{Histologic Examination and TMA Construction}

Hematoxylin and eosin (H\&E)-stained slides were reviewed for confirmation of histopathologic diagnoses and selection of adequate specimens for analysis. All cases were classified according to the histological classification of lung tumors specified by the World Health Organization. ${ }^{15}$ Pathologic stage was based on the revised international system. ${ }^{16}$ The diagnoses were established by examination of conventional H\&E-stained slides for most cases. Neutral buffered formalin-fixed $(10 \% \mathrm{vol} /$ formalin in water; pH: 7.4) and paraffin-embedded tissue blocks were retrieved from the Department of Pathology, Paraffin Archive Resource of Roswell Park Cancer Institute. Areas of viable tumor as well as normal tissue elements were identified and marked by an investigator (DFT) for the construction of microarrays. High-density TMAs were assembled using a Beecher tissue puncher/array system (Beecher Instruments, Silver Spring, MD, USA) as previously described. ${ }^{17}$ The system consists of thin-walled stainless-steel needles with an inner diameter of approximately $1 \mathrm{~mm}$ and a stylet for transferring and emptying the needle contents. The assembly is held in an X-Y position guide that is manually adjusted by digital micrometers. Specimens were retrieved from selected regions of the donor paraffin block and are precisely arrayed in a new recipient block. Tissue cores were $1.0 \mathrm{~mm}$ in diameter and ranged in length from 1.0 to $3.0 \mathrm{~mm}$ depending on the depth of tissue available in the donor block. For each case, two to three core samples of normal and tumor tissue were acquired from two different donor blocks resulting in 336 cores. Cores were assembled in three highdensity TMA blocks. One arrayed core contained up to 3200 neoplastic cells.

\section{IHC and Scoring}

Sections, 5 - $\mu$ m-thick, were cut from microarrays for IHC and processed within 1 week of cutting to avoid oxidation of antigens. Initial sections were stained for H\&E to verify histology. Avidin-biotin-staining method was employed as previously described. ${ }^{14}$ Briefly, for E-cad, antigen retrieval was carried out using Dako Target Retrieval Solution (H1, Target Retrieval Solution, pH: 9.9, 10 min, Dako, CA, USA). Mouse monoclonal antibodies against E-cad (clone: 36B5, Novocastra, UK) were used at a dilution of 1:50. These antibodies react with the N-terminal external region of E-cad. For EGFR, the sections 
were treated with proteinase K (Dako, CA, USA) for 5 min. Mouse monoclonal antibodies against EGFR (clone: H11, Dako, CA, USA) were used at a dilution of 1:200. These antibodies react with $\mathrm{M}_{\mathrm{r}} 170000$ wild-type EGFR and the Mr 145000 deletion mutant form of the receptor (EGFRvIII). The antibodies were incubated with the array sections for $30 \mathrm{~min}$ at room temperature in an automatic immuostainer (Dako, CA, USA), and then with a detection kit in accordance with the manufacturer's instructions. Immunoreactivity of the normal lung tissue was used as a positive control. Two investigators (DFT, GD) independently reviewed the IHC slides without the knowledge of patient outcome. The percentages of stained neoplastic cells in TMA sections were assessed for each case, as well as the pattern of their staining. More than 10\% unequivocal membrane staining was considered a cut-off point for positive E-cad and EGFR expression. In addition, E-cad and EGFR cytoplasmic pattern of staining were reported separately.

\section{Statistical Analysis}

For statistical analyses, patients were separated according to E-cad expression into positive $(+)$ and negative $(-)$ groups; the latter group included tumors with only cytoplasmic expression. Regarding E-cad and EGFR coexpressions, the patients were divided into: E-cad(-)/EGFR(-), E-cad(-)/EGFR $(+)$, E-cad $(+) / \operatorname{EGFR}(-)$, and E-cad $(+) / \operatorname{EGFR}(+)$ groups. In addition, one group with only cytoplasmic expression of E-cad was analyzed separately. Categorical variables were analyzed using Fisher's exact test. The interobserver reproducibility was evaluated by analysis of Spearman's rank test. Age was analyzed using the Mann-Whitney rank-sum test. The follow-up time was calculated using the potential follow-up method. The overall patient survival was defined as the time between the date of surgical diagnosis to the date of last follow-up (censored) or date of patient death (event). Differences in survival times between patient subgroups were analyzed using the log-rank test and proportional hazard regression model. Survival probabilities were estimated using the KaplanMeier method. ${ }^{18}$ Statistical significance for model parameters was based on the likelihood ratio test. Cox proportional hazards regression analysis with SAS software (Statistical Analysis Systems) was used to determine the association between clinicopathologic variables and overall patient survival. In all tests, statistical significance was set at $P<0.05$.

\section{Results}

\section{Clinicopathologic Data}

After immunohistochemical staining, 118, 126, and 115 cases were fully assessable for E-cad, EGFR, and both markers, respectively. In the E-cad group, there were $55(47 \%)$ women and $63(53 \%)$ men with a median age of 65.2 years. NSCLC histologic subtypes were $81(69 \%)$ AdCs and 37 (31\%) SCCs. These tumors were 10 (8\%) well-differentiated, 41 (35\%) moderately differentiated, and 67 (57\%) poorly differentiated. There were 97 tumors $(82 \%)$ with stage I and $21(18 \%)$ with stages II or IIIA. In all, 79 patients $(67 \%)$ had negative lymph node metastases, where 39 (33\%) had one or two positive lymph nodes. Regarding EGFR group, there were 57 $(45 \%)$ women and $69(55 \%)$ men, with a median age of 65.0 years. NSCLC histologic subtypes were 83 (66\%) AdCs and 43 (34\%) SCCs. These tumors were $10(8 \%)$ well-differentiated, $43(34 \%)$ moderately differentiated, and 73 (58\%) poorly differentiated. There were 102 patients $(81 \%)$ with stage I disease and $24(19 \%)$ with stages II or IIIA disease. In all, 85 patients $(67 \%)$ had negative lymph nodes, where 41 $(32 \%)$ had one or two positive lymph nodes. The combined E-cad and EGFR groups had 54 (47\%) women and $61(53 \%)$ men with a median age of 65.3 years. NSCLC histologic subtypes were $78(68 \%)$ AdCs and $37(32 \%)$ SCCs. These tumors were 10 (9\%) well-differentiated, 39 (34\%) moderately differentiated, and $66(57 \%)$ poorly differentiated. There were 94 patients (82\%) with stage I disease and $21(18 \%)$ with stages II or IIIA disease. A total of 79 patients $(67 \%)$ had no evidence of lymph node metastases and $38(33 \%)$ had one or two pathologically positive lymph nodes. Patients' clinicopathologic features, including those with only cytoplasmic E-cad expression, are summarized in Table 1.

\section{Expression of E-cad and EGFR in Normal Lung Tissue and in Adc and SCC}

The normal respiratory and alveolar epithelia exhibited a delicate membrane staining for E-cad (Figure 1a and b). Cytoplasmic staining alone was not observed in the normal epithelium. The respiratory epithelium had EGFR membrane expression with basal localization. The alveolar epithelium was negative for this marker (Figure 1c and d).

The immunohistochemical data of E-cad and EGFR expressions in assessable tumor cases are shown in Tables 2 and 3 . The neoplastic cells in the TMA sections had a delicate membrane expression of E-cad and EGFR. In all, 53 tumors (45\%) were E-cad(-) and $65(55 \%)$ were $(+)$. According to the histologic types, 47/81 cases (58\%) of AdCs and 18/ $37(49 \%)$ of SCCs were E-cad $(+)$. A total of 23 tumors $(19 \%)$ had only cytoplasmic expression of E-cad; 14/23 tumors (61\%) were AdCs and 9/23 $(39 \%)$ were SCCs. In all, 43 tumors $(34 \%)$ were EGFR $(+)$ and $83(66 \%)$ were $(-)$. Out of the 83 cases $18(22 \%)$ of AdCs and 25/43 (58\%) of SCCs were $\operatorname{EGFR}(+)$. The only cytoplasmic pattern of staining 
Table 1 Clinicopathologic features of the study group

\begin{tabular}{|c|c|c|c|c|c|}
\hline Variables & & $\begin{array}{c}E \text {-cad } \\
(\mathrm{n}=118)\end{array}$ & $\begin{array}{c}E-\operatorname{cad}(c)^{1} \\
(\mathrm{n}=23)\end{array}$ & $\begin{array}{c}E G F R \\
(\mathrm{n}=126)\end{array}$ & $\begin{array}{c}\text { E-cad and EGFR } \\
\quad(\mathrm{n}=115)\end{array}$ \\
\hline Age (years) & Median & 65.2 & 67.2 & 65 & 65.3 \\
\hline \multirow[t]{2}{*}{ Sex } & Female & $55(47 \%)$ & $10(43 \%)$ & $57(45 \%)$ & $54(47 \%)$ \\
\hline & Male & $63(53 \%)$ & $13(57 \%)$ & $69(55 \%)$ & $61(53 \%)$ \\
\hline \multirow[t]{2}{*}{ Smoke $^{\mathrm{a}}$} & Never & $12(10 \%)$ & $1(5 \%)$ & $13(10 \%)$ & $12(11 \%)$ \\
\hline & Ever & $105(90 \%)$ & $21(95 \%)$ & $112(90 \%)$ & $102(89 \%)$ \\
\hline \multirow[t]{2}{*}{$\mathrm{PS}^{\mathrm{a}}$} & 0 & $79(68 \%)$ & $15(68 \%)$ & $86(69 \%)$ & $77(68 \%)$ \\
\hline & $1,2,3$ & $38(32 \%)$ & $7(32 \%)$ & 39 (31\%) & $37(32 \%)$ \\
\hline \multirow[t]{2}{*}{$W^{b}$} & Absent & $104(90 \%)$ & $20(91 \%)$ & $109(88 \%)$ & $101(89 \%)$ \\
\hline & Present & $12(10 \%)$ & 2 (9\%) & $15(12 \%)$ & $12(11 \%)$ \\
\hline \multirow[t]{2}{*}{ Histology } & AdC & $81(69 \%)$ & $14(61 \%)$ & $83(66 \%)$ & $78(68 \%)$ \\
\hline & SCC & $37(31 \%)$ & $9(39 \%)$ & $43(34 \%)$ & $37(32 \%)$ \\
\hline \multirow[t]{3}{*}{ Grade } & Well & $10(8 \%)$ & 0 & $10(8 \%)$ & $10(9 \%)$ \\
\hline & Mod & $41(35 \%)$ & $6(26 \%)$ & $43(34 \%)$ & $39(34 \%)$ \\
\hline & Poor & $67(57 \%)$ & $17(74 \%)$ & $73(58 \%)$ & $66(57 \%)$ \\
\hline \multirow[t]{2}{*}{ Stage } & I & $97(82 \%)$ & $18(78 \%)$ & $102(81 \%)$ & $94(82 \%)$ \\
\hline & II, IIIA & $21(18 \%)$ & $5(21 \%)$ & $24(19 \%)$ & $21(18 \%)$ \\
\hline \multirow[t]{2}{*}{$\mathrm{PN}$} & Negative & $79(67 \%)$ & $16(70 \%)$ & $85(67 \%)$ & $77(67 \%)$ \\
\hline & Positive & $39(33 \%)$ & $7(30 \%)$ & $41(32 \%)$ & $38(33 \%)$ \\
\hline
\end{tabular}

c, cytoplasmic staining only; PS, performance status; WL, weight loss. AdC, adenocarcinoma; SCC, squamous cell carcinoma. Well, well differentiated; mod, moderately differentiated; poor, poorly differentiated. PN, lymph node metastasis.

${ }^{\mathrm{a}}$ One patient lacked smoking and PS data.

$\mathrm{b}_{\text {Two patients lacked WL data. }}$

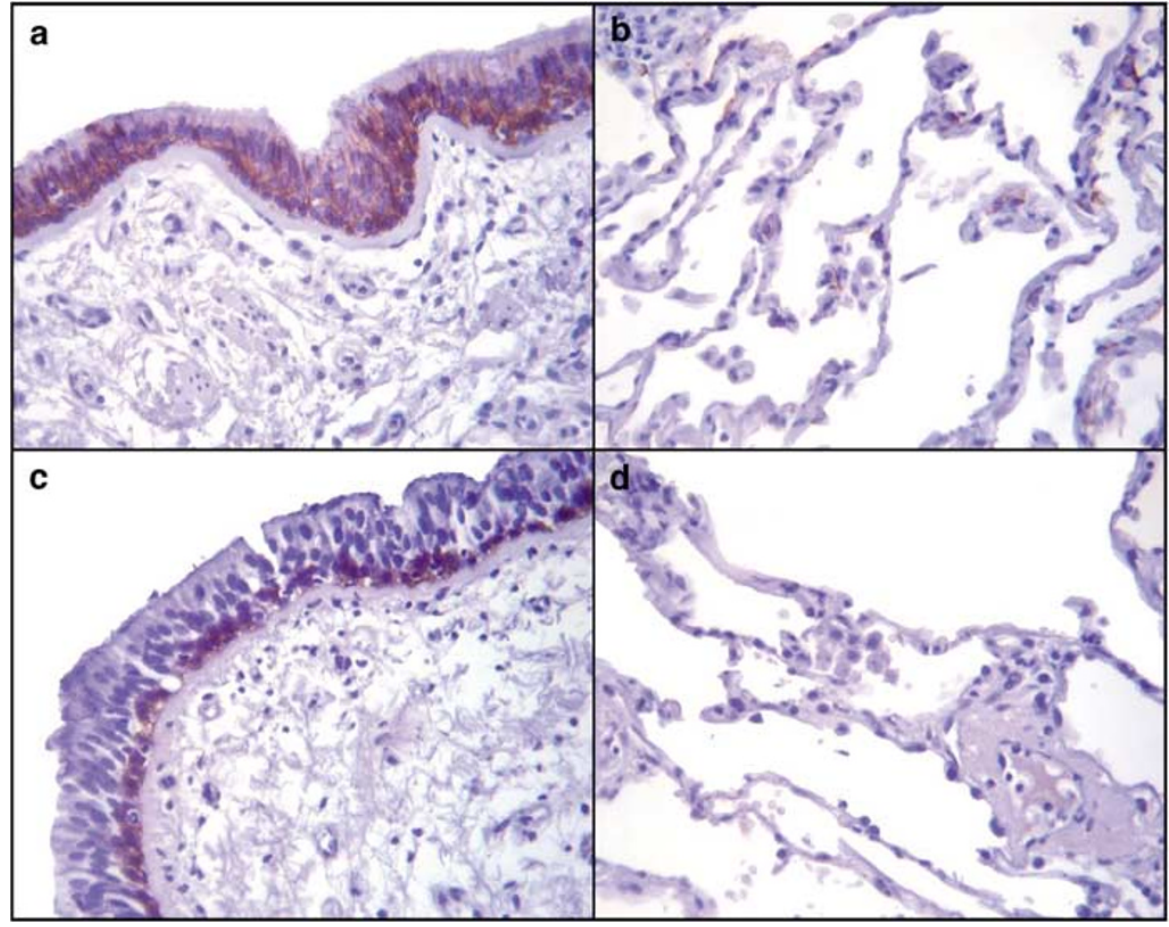

Figure 1 (a) and (b) Benign respiratory and alveolar epithelia, respectively, show a membrane-staining pattern of E-cadherin (E-cad) expression. (c) Benign respiratory epithelium basal layer shows a membrane-staining pattern of epidrmal growth factor receptor (EGFR) expression in contrast to the alveolar lining (d) which is negative for EGFR expression (immunoperoxidase stain, $\times 200$ ).

was not identified in $\operatorname{EGFR}(+)$ cases. $\operatorname{EGFR}(+)$ SCCs, when compared with the normal respiratory epithelium, showed a pattern of staining that extended beyond the basal layer to involve variable portions of the neoplastic epithelial thickness. Both markers were simultaneously $(+)$ in $13 / 115$ cases $(11 \%)$ and were $(-)$ in $22 / 115(19 \%)$. There were $52 /$ 115 cases (44\%) with E-cad(+)/EGFR(-) and 28/ $115(24 \%)$ with E-cad(-)/EGFR(+). When stratifying markers' expressions according to the tumor 
grade, EGFR(+) was more frequent in poorly differentiated than in well and moderatly differentiated AdCs. Out of the 48 cases 14 (29\%) of poorly differentiated AdCs were $(+)$ for EGFR, where 1/9 $(11 \%)$ and $3 / 26(12 \%)$ of well and moderately differentiated AdCs, respectively, were $(+)$ for the latter marker. The only cytoplasmic expression of Ecad was observed exclusively in both moderately and poorly differentiated AdC and SCC, and it was more frequent in poorly than in moderately differentiated tumors. Detailed expressions of E-cad and EGFR in tumor grade strata is shown in Tables 2 and 3. Representative photomicrographs of E-cad and EGFR expressions in tumor samples are shown in (Figure 2a-d).

\section{Association of E-cad and EGFR with Clinicopathologic Parameters and Overall Survival}

There was no statistically significant association between E-cad and any of the clinicopathologic parameters analyzed. This was also true for EGFR and for simultaneous assessment of both markers; the only exception was the association between
$\operatorname{EGFR}(+)$ and SCC histologic type $(P<0.001$, Fisher's exact test). Among the analyzed clinicopathologic parameters only performance status, stage, lymph node involvement, and age were significantly associated with patient survival in both univariate and multivariate analyses (Table 4). Patients with E-cad positive tumors had longer overall survival when compared to those with negative expression $(P=0.008$, log-rank test) (Figure 3). Interestingly, patients with only cytoplasmic expression of E-cad had a shorter survival in comparison with those who had positive expression $(P=0.002$, log-rank test $)$ (Figure 4). In multivariate analysis, E-cad $(+)$ patients were still having a longer survival $(P=0.016)$ than those with negative and/or cytoplasmic staining. There is no statistically significant difference in survival between EGFR $(+)$ patients and those who were negative for this marker $(P>0.05)$. Analyzing coexpression of E-cad and EGFR after dividing our patients into four groups indicated an association between E-cad(-)/EGFR $(+)$ phenotype and worse patient survival $(P=0.026$, log-rank test) (Figure 5) in comparison with E-cad( + )/EGFR( $(-)$ phenotype. However, this association was not valid in multivariate analysis $(P>0.05)$. The association between

Table 2 E-cad and EGFR expressions in lung AdC and SCC

\begin{tabular}{|c|c|c|c|c|c|c|c|}
\hline \multirow[t]{2}{*}{ Grade } & \multicolumn{2}{|c|}{$E-\operatorname{cad}(-)$} & \multirow{2}{*}{$\begin{array}{l}E-\operatorname{cad}(+) \\
(\mathrm{n}=65)\end{array}$} & \multirow{2}{*}{$\begin{array}{c}\text { Total } \\
(\mathrm{n}=118)\end{array}$} & \multirow{2}{*}{$\begin{array}{l}\operatorname{EGFR(-)} \\
(\mathrm{n}=83)^{a}\end{array}$} & \multirow{2}{*}{$\begin{array}{l}\text { EGFR(+) } \\
(\mathrm{n}=43)\end{array}$} & \multirow{2}{*}{$\begin{array}{c}\text { Total } \\
(\mathrm{n}=126)\end{array}$} \\
\hline & $(\mathrm{n}=30)^{a}$ & $(\mathrm{n}=23)^{b}$ & & & & & \\
\hline$A d C$ & $20(25 \%)$ & $14(17 \%)$ & 47 (58\%) & $81(100 \%)$ & 65 (78\%) & $18(22 \%)$ & $83(100 \%)$ \\
\hline Well & $4(44 \%)$ & 0 & $5(56 \%)$ & 9 & $8(89 \%)$ & $1(11 \%)$ & 9 \\
\hline Mod & $5(20 \%)$ & $4(16 \%)$ & $16(64 \%)$ & 25 & $23(88 \%)$ & $3(12 \%)$ & 26 \\
\hline Poor & $11(24 \%)$ & $10(21 \%)$ & $26(55 \%)$ & 47 & $34(71 \%)$ & $14(29 \%)$ & 48 \\
\hline$S C C$ & $10(27 \%)$ & $9(24 \%)$ & $18(49 \%)$ & 37 (100\%) & $18(42 \%)$ & 25 (58\%) & $43(100 \%)$ \\
\hline Well & 0 & 0 & 1 & 1 & 0 & 1 & 1 \\
\hline Mod & $7(44 \%)$ & $2(12 \%)$ & 7 (44\%) & 16 & $7(41 \%)$ & $10(59 \%)$ & 17 \\
\hline Poor & $3(15 \%)$ & $7(35 \%)$ & $10(50 \%)$ & 20 & $11(44 \%)$ & $14(56 \%)$ & 25 \\
\hline
\end{tabular}

AdC, adenocarcinoma; well, well differentiated; mod, moderately differentiated; poor, poorly differentiated. SCC, squamous cell carcinoma.

${ }^{\mathrm{a}}$ Cases with no and less than $10 \%$ staining.

${ }^{\mathrm{b}}$ Cases with only cytoplasmic staining.

Table 3 E-cad and EGFR coexpressions in lung AdC and SCC

\begin{tabular}{|c|c|c|c|c|c|}
\hline Grade & $\begin{array}{c}E-\operatorname{cad}(-) / E G F R(-) \\
(\mathrm{n}=22)\end{array}$ & $\begin{array}{c}E-\operatorname{cad}(-) / E G F R(+) \\
(\mathrm{n}=28)\end{array}$ & $\begin{array}{c}E-\operatorname{cad}(+) / E G F R(-) \\
(\mathrm{n}=52)\end{array}$ & $\begin{array}{c}E-\operatorname{cad}(+) / E G F R(+) \\
(\mathrm{n}=13)\end{array}$ & $\begin{array}{c}\text { Total } \\
(\mathrm{n}=115)\end{array}$ \\
\hline$A d C$ & $19(24 \%)$ & $12(15 \%)$ & $41(53 \%)$ & $6(8 \%)$ & $78(100 \%)$ \\
\hline Well & $3(33 \%)$ & $1(11 \%)$ & $5(56 \%)$ & 0 & 9 \\
\hline Mod & $5(22 \%)$ & $2(9 \%)$ & $15(65 \%)$ & $1(4 \%)$ & 23 \\
\hline Poor & $11(24 \%)$ & $9(20 \%)$ & $21(46 \%)$ & $5(10 \%)$ & 46 \\
\hline$S C C$ & $3(8 \%)$ & $16(43 \%)$ & $11(30 \%)$ & 7 (19\%) & 37 (100\%) \\
\hline Well & 0 & 0 & 0 & 1 & 1 \\
\hline Mod & $2(12 \%)$ & 7 (44\%) & $4(25 \%)$ & 3 (19\%) & 16 \\
\hline Poor & $1(5 \%)$ & $9(45 \%)$ & 7 (35\%) & $3(15 \%)$ & 20 \\
\hline
\end{tabular}

AdC, adenocarcinoma; well, well differentiated; mod, moderately differentiated; poor, poorly differentiated. SCC, squamous cell carcinoma. 


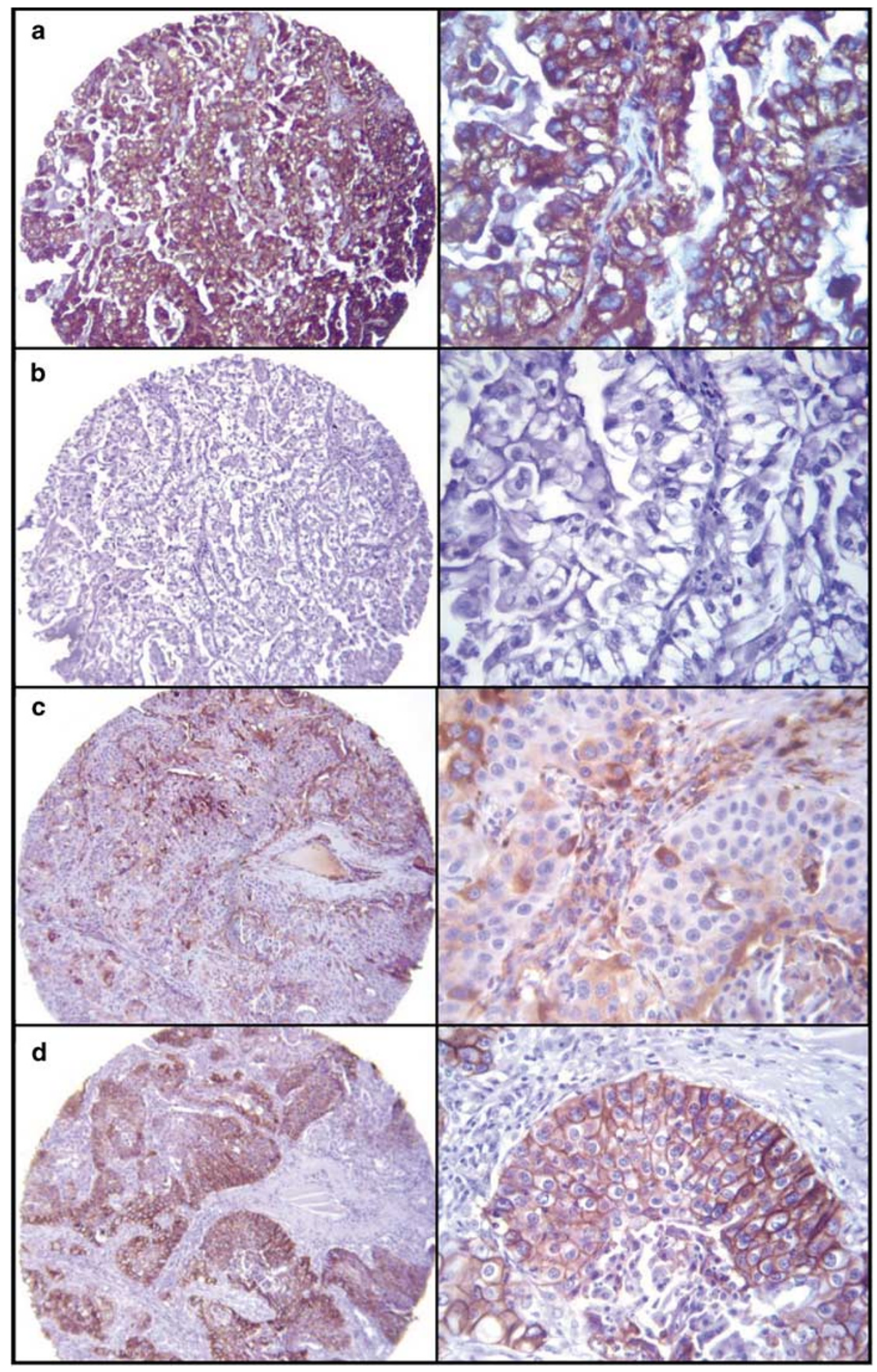

Figure 2 A case of adenocarcinoma (AdC), which is diffusely positive ( $>90 \%$ ) for membrane E-cad (a), but otherwise is negative for EGFR in the same section profile (b). A case of squamous cell carcinoma, (SCC) which is negative for membrane E-cad, but shows only cytoplasmic staining for this marker (c). The same case, in the same section profile, is $>90 \%$ positive for membrane EGFR (d) (immunoperoxidase staining, $\times 100$ and $\times 400$.

E-cad and EGFR expressions and patient survival is shown in Table 4.

\section{Discussion}

Identifying molecular markers to distinguish resectable NSCLC patients with a high risk of recurrence is crucial to improving therapeutic outcome. Therefore, several biological effectors related to cell adhesions, growth, and differentiation have been studied in individuals who develop NSCLC. In this study, we utilized a TMA method to screen a group of NSCLC for the expression of E-cad and EGFR independently and simultaneously and for evaluating their role as prognosticators. TMA is a procedure 
Table 4 Association of E-cad, EGFR, and both with survival: univariate and multivariate analyses

\begin{tabular}{|c|c|c|c|c|}
\hline \multicolumn{2}{|l|}{ Variables } & Relative risk & $95 \% C I$ & $\mathrm{P}$-value \\
\hline \multicolumn{5}{|c|}{ Univariate analysis $^{a}$} \\
\hline \multirow[t]{2}{*}{ Age (years) } & Median & Ref & & \\
\hline & $>$ Median & 1.2 & $1.1-3.0$ & 0.025 \\
\hline \multirow[t]{2}{*}{ PS } & 0 & Ref & & \\
\hline & $1,2,3$ & 3.3 & $2.0-5.5$ & $<0.001$ \\
\hline \multirow[t]{2}{*}{ Stage } & I & Ref & & \\
\hline & II, IIIA & 2.9 & $1.7-5.1$ & $<0.001$ \\
\hline \multirow[t]{2}{*}{$\mathrm{PN}$} & Negative & Ref & & \\
\hline & Positive & 2.5 & $1.5-4.1$ & $<0.001$ \\
\hline \multirow[t]{2}{*}{ E-cad } & Negative & Ref & & \\
\hline & Positive & 0.5 & $0.3-0.8$ & 0.008 \\
\hline \multirow{2}{*}{ E-cad(c) } & Absent & Ref & & \\
\hline & Present & 0.4 & $0.2-0.7$ & 0.002 \\
\hline \multirow[t]{2}{*}{ EGFR } & Negative & Ref & & \\
\hline & Positive & 1.5 & $0.9-2.5$ & $>0.05$ \\
\hline \multirow[t]{2}{*}{ E-cad/EGFR } & $(-/+)$ & Ref & & \\
\hline & $(+/-)$ & 2.1 & $1.1-4.0$ & 0.026 \\
\hline \multicolumn{5}{|c|}{ Multivariate analysis ${ }^{a}$} \\
\hline \multirow[t]{2}{*}{ Age (years) } & Median & Ref & & \\
\hline & $>$ Median & 1.9 & $1.1-3.3$ & 0.032 \\
\hline \multirow[t]{2}{*}{ PS } & 0 & Ref & & \\
\hline & $1,2,3$ & 3.1 & $1.8-5.4$ & $<0.001$ \\
\hline \multirow[t]{2}{*}{ Stage } & I & Ref & & \\
\hline & II, IIIA & 2.3 & $1.0-5.2$ & 0.042 \\
\hline \multirow[t]{2}{*}{$\mathrm{PN}$} & Negative & Ref & & \\
\hline & Positive & 2.0 & $1.0-4.1$ & 0.061 \\
\hline \multirow[t]{2}{*}{ E-cad } & Negative & Ref & & \\
\hline & Positive & 0.5 & $0.3-0.9$ & 0.016 \\
\hline \multirow[t]{2}{*}{ E-cad(c) } & Absent & Ref & & \\
\hline & Present & 0.4 & $0.2-0.8$ & 0.007 \\
\hline \multirow[t]{2}{*}{ EGFR } & Negative & Ref & & \\
\hline & Positive & 1.1 & $0.6-2.0$ & $>0.05$ \\
\hline \multirow[t]{2}{*}{ E-cad/EGFR } & $(-/+)$ & Ref & & \\
\hline & $(+/-)$ & 1.8 & $0.8-3.8$ & $>0.05$ \\
\hline
\end{tabular}

PS, performance status; PN, lymph node metastasis; c, cytoplasmic staining only.

${ }^{\mathrm{a} C l i n i c o p a t h o l o g i c ~ v a r i a b l e s ~(a g e, ~ P S, ~ s t a g e, ~ a n d ~ P N) ~ a r e ~ t h o s e ~ o f ~ t h e ~}$ largest group (EGFR group, $n=126$ ).

that has proven to be a valid methodology in screening human tumor tissue for different biological markers. ${ }^{19}$ Our selected group of patients was validated by integrating newly studied markers with significant factors identified in earlier studies, such as tumor lymph node metastasis (TNM) staging system parameters. ${ }^{16,20}$ The stage of disease and the lymph node involvement were correlated with patient survival in both univariate and multivariate statistical analyses, a fact that supports the accurate representation of our TMA samples.

Lee et $a l^{9}$ studied 115 cases of NSCLC $(3 \mathrm{~cm}$ or less in size) for E-cad and catenins expressions using IHC. They found that membranous E-cad expression was associated with tumor dedifferentiation, invasiveness, and advanced stage, although it did not have a prognostic value in multivariate analysis. Kase et $a l^{21}$ assessed the expression of E-cad and $\beta$-catenin in 331 cases of NSCLC using IHC. They concluded that there was no

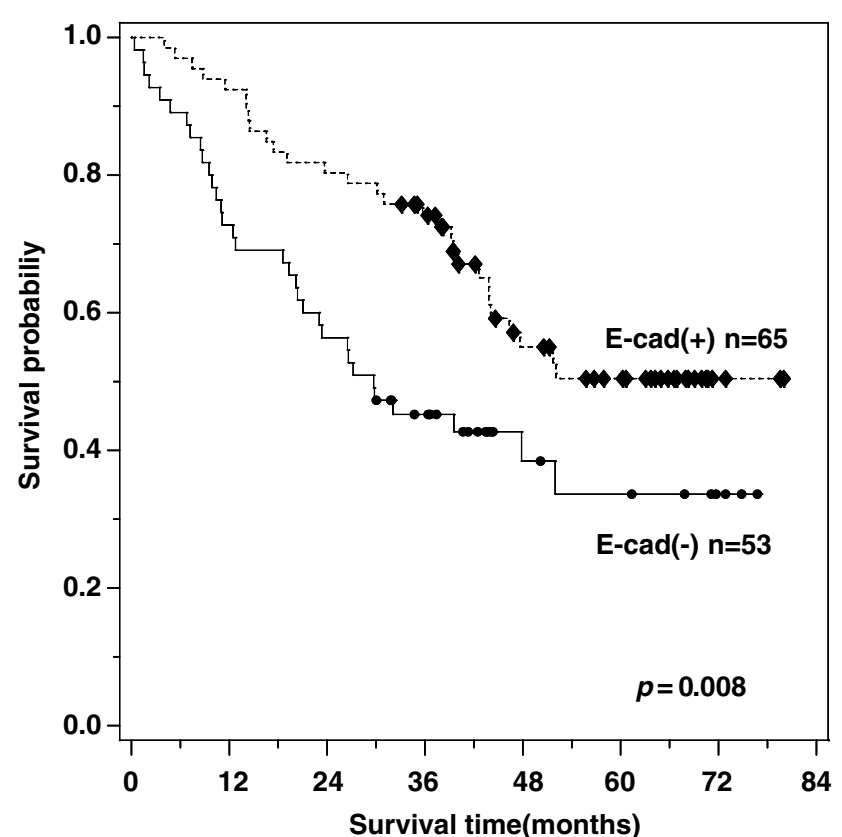

Figure 3 Survival curve of lung cancer (AdC and SCC) patients according to E-cad expression: positive membrane staining with a median survival time of 56.1 months vs negative staining with a median survival time of 40 months $(P=0.008$, log-rank test).

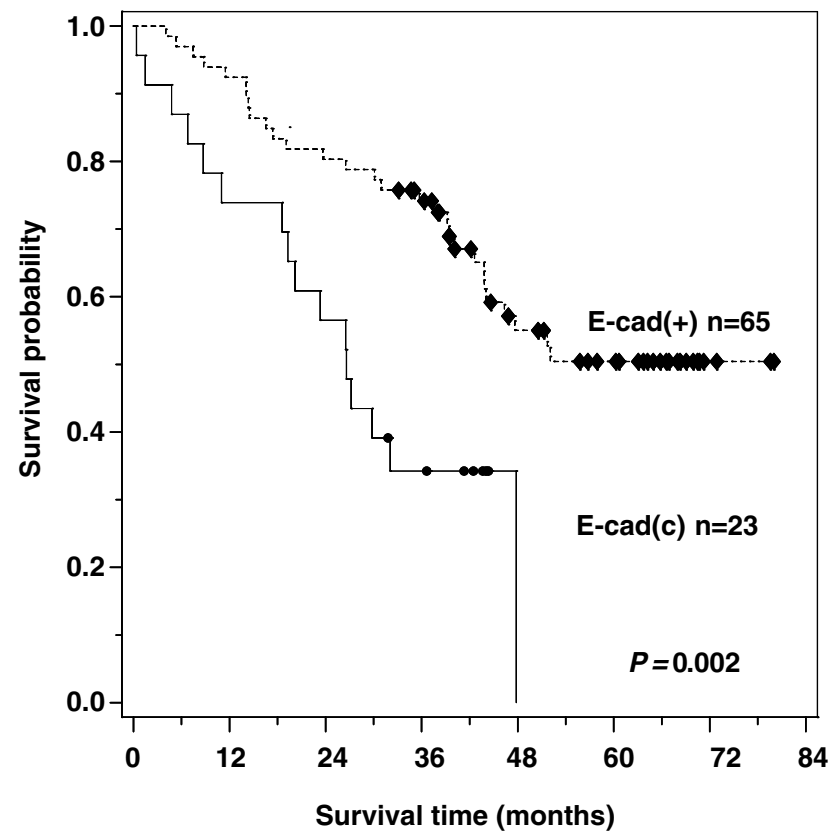

Figure 4 Survival curve of lung cancer (AdC and SCC) patients according to E-cad expression: positive membrane staining with a median survival time of 56.1 months vs cytoplasmic staining (c) with a median survival time of 30.4 months $(P=0.002$, log-rank test).

independent predictive value of E-cad as a disease prognosticator; only reduced expression of both membrane E-cad and $\beta$-catenin expressions was 


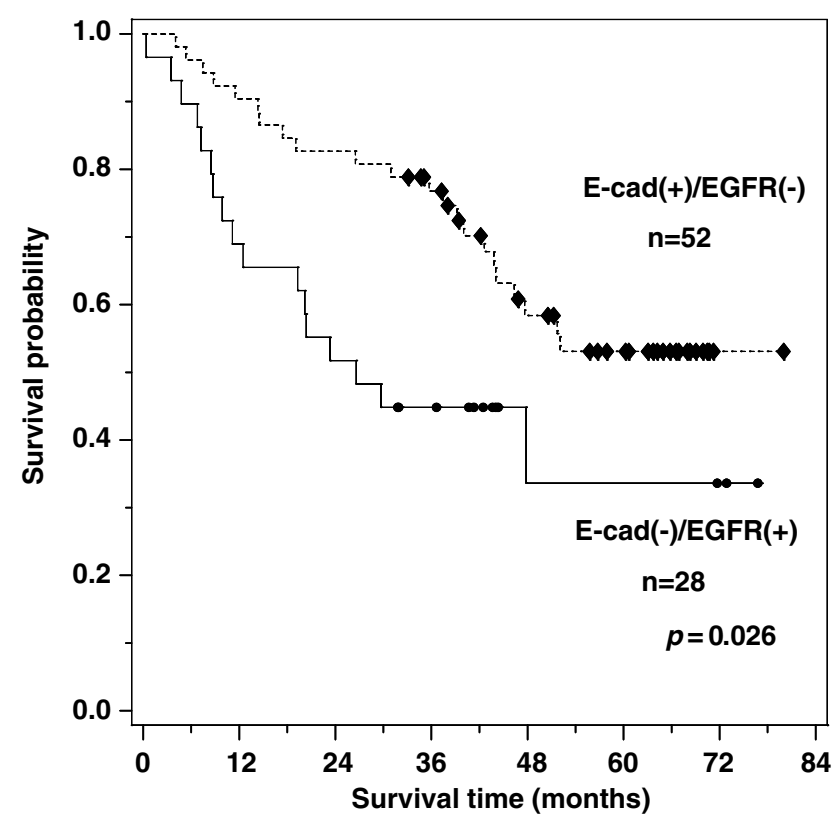

Figure 5 Survival curve of lung cancer (AdC and SCC) patients according to E-cad and EGFR coexpressions: positive E-cad/ EGFR(-) group with a median survival time of 57.7 months vs negative E-cad/EGFR $(+)$ group with a median survival time of 41.4 months $(P=0.026$, log-rank test $)$.

significantly correlated with a poor prognosis $(P=0.0003)$.

In contrast, our results support that E-cad expression using IHC, when taking into account the unequivocal membrane staining in $>10 \%$ of neoplastic cells, is statistically associated with favorable patient survival in univariate and multivariate analyses $(P=0.008$ and 0.016 , respectively). This finding is also in concordance with a recent report by Bremnes et $a l^{22}$ The authors utilized TMA and IHC to evaluate E-cad pathway cofactors. They showed that membranous E-cad expression is an independent prognostic factor for NSCLC patient survival $(P=0.002)$. However, their study did not comment on the significance of cytoplasmic expression of E-cad in relation to survival. In our study, we observed a cytoplasmic staining of E-cad in a minor subset of NSCLC (Figure 2c); the pattern that may be suggestive of cytoplasmic localization of this protein. Interestingly, cytoplasmic staining of E-cad was associated with shorter patient survival in both univariate and multivariate analyses $(P=0.002$ and 0.007 , respectively). In addition the absence of E-cad cytoplasmic expression from benign pulmonary epithelia and strict expression in malignant epithelia may be a tumorigenesis-related phenomenon. Bremnes et $a l^{22}$ observed nuclear staining of E-cad only when using antibodies that react to the cytoplasmic domain of the molecule. The antibodies we used recognize the N-terminal external region of E-cad. Therefore, E-cad cytoplasmic staining may be due to E-cad proteolytic cleavage by a membrane- bound metalloprotease to yield a soluble form; ${ }^{23}$ this mechanism is referred to as 'regulated intramembranous proteolysis'. ${ }^{24}$ However, a defective intracellular transportation pathway, or a nonspecific reaction of E-cad antibodies with intracytoplasmic proteins may also account for this observation. Although nuclear staining of E-cad protein has been described in association with Merkel cell tumors of the skin, ${ }^{25}$ we did not observe such an expression in our NSCLC patient cohort. The reason for this difference may be the different antibodies or retrieval methods used.

EGFR has a complex role in cell transduction signaling that promotes cell proliferation and differentiation. ${ }^{5}$ Therefore, targeting the EGFR tyrosine kinase activity might represent an effective therapeutic modality for the treatment of NSCLC patients. $^{26,27}$ The existing literature regarding EGFR expression in NSCLC and its association with patient survival has been inconsistent. Nicholson et $a l,{ }^{10}$ after reviewing the literature from 1985 to 2000 regarding EGFR and cancer types association, indicated that this marker was rarely related to other clinical and prognostic markers in NSCLC. However other studies had reported a relationship between EGFR expression and patient survival, ${ }^{28}$ as well as a relationship between this expression and tumor pathogenesis progression. ${ }^{29}$

In our cohort, there was no statistical correlation between diffuse membranous expression of EGFR and patient survival $(P>0.05)$. However, all EGFR $(+)$ tumors, markedly SCCs, had a neoplastic distribution of the marker beyond its basal localization in benign respiratory epithelium; this finding, which was also observed by others, ${ }^{29}$ suggests that a correlation between diffuse EGFR expression and tumor progression may exist.

Although both markers, E-cad and EGFR, have been individually studied in NSCLC, assessment of their coexpression and its relationship to patient survival has been limited and inconsistent. Sorscher et $a l^{13}$ evaluated the expression of E-cad and EGFR in 37 cases of stages I and II NSCLC. The authors used IHC to assess the nuclear and cytoplasmic staining of both markers. They found a statistically significant correlation between E-cad and EGFR staining in primary tumor, but not between the primary tumor and its metastatic disease.

In the current study, we divided our cases into four groups: $\operatorname{E-cad}(-) / \operatorname{EGFR}(-), \operatorname{E-cad}(-) / \operatorname{EGFR}(+)$, $\operatorname{E-cad}(+) / \operatorname{EGFR}(-)$, and $\operatorname{E-cad}(+) / \operatorname{EGFR}(+)$. There was a significant difference in patient survival between E-cad $(-) / \operatorname{EGFR}(+)$ group and E-cad $(+) /$ EGFR(-) group, with the former associated with shorter patient survival $(P=0.026)$ (Figure 5). Although this result is in agreement with a previously reported correlation between E-cad $(+) /$ EGFR(-) tumor phenotype and longer patient survival in esophageal cancer, $^{30}$ the association failed the multivariate statistical analysis. The latter statistical failure might be due to the small study 
size and/or multiple parameters used; nevertheless, because of the hypothesized complicated biological relationship between the two markers, a large prospective study may be needed to further assess this relation at an immunohistochemical observational level.

No significant association between E-cad and EGFR expressions and tumor differentiation was entertained in these strictly selected cases of resectable (stages I-IIIA) AdCs and SCCs; however, an increased frequency of EGFR expression was seen in poorly differentiated AdCs in comparison with more differentiated ones (Table 2).

In conclusion, TMA is a feasible and useful procedure for screening NSCLC for E-cad and EGFR protein expressions. Decreased membranous expression of E-cad is associated with shorter patient survival. Owing to of its association with unfavorable disease outcome, the only cytoplasmic expression of E-cad may represent an intermediate level of E-cad intracytoplasmic degradation in NSCLC. Although it did not associate with patient survival, diffuse EGFR expression may be related to tumor progression. Our study suggests that NSCLC may be grouped based on the expression of E-cad and EGFR with E-cad(-)/EGFR(+) expression having a probable worse disease prognosis. Evaluation of E-cad and EGFR may represent an important disease prognosticator and may aid in clinical decision-making with respect to the therapy employed.

\section{References}

1 D’Amico TA, Massey M, Herndon II JE, et al. A biologic risk model for stage I lung cancer: immunohistochemical analysis of 408 patients with the use of ten molecular markers. J Thorac Cardiovasc Surg 1999;117: 736-743.

2 O’Byrne KJ, Cox G, Swinson D, et al. Towards a biological staging model for operable non-small cell lung cancer. Lung Cancer 2001;34(Suppl 2):S83-S89.

3 Takeichi M. Cadherins: a molecular family important in selective cell-cell adhesion. Annu Rev Biochem 1990;59:237-252.

4 Takeichi M. Cadherin cell adhesion receptors as a morphogenetic regulator. Science 1991;251:14511455.

5 Wells A. EGF receptor. Int J Biochem Cell Biol 1999;31:637-643.

6 Jawhari AU, Farthing MJ, Pignatelli M. The E-cadherin/epidermal growth factor receptor interaction: a hypothesis of reciprocal and reversible control of intercellular adhesion and cell proliferation. J Pathol 1999;187:155-157.

7 Shiozaki H, Kadowaki T, Doki Y, et al. Effect of epidermal growth factor on cadherin-mediated adhesion in a human oesophageal cancer cell line. Br J Cancer 1995;71:250-258.

8 al Moustafa AE, Alaoui-Jamali M, Paterson J, et al. Expression of P185erbB-2, P160erbB-3, P180erbB-4, and heregulin alpha in human normal bronchial epithelial and lung cancer cell lines. Anticancer Res 1999;19:481-486.

9 Lee YC, Wu CT, Chen CS, et al. The significance of E-cadherin and alpha-, beta-, and gamma-catenin expression in surgically treated non-small cell lung cancers of $3 \mathrm{~cm}$ or less in size. J Thorac Cardiovasc Surg 2002;123:502-507.

10 Nicholson RI, Gee JM, Harper ME. EGFR and cancer prognosis. Eur J Cancer 2001;37(Suppl 4):S9-S15.

11 Fontanini G, Vignati S, Bigini D, et al. Epidermal growth factor receptor (EGFr) expression in non-small cell lung carcinomas correlates with metastatic involvement of hilar and mediastinal lymph nodes in the squamous subtype. Eur J Cancer 1995;31A: 178-183.

12 Ohsaki Y, Tanno S, Fujita Y, et al. Epidermal growth factor receptor expression correlates with poor prognosis in non-small cell lung cancer patients with p53 overexpression. Oncol Rep 2000;7:603-607.

13 Sorscher SM, Russack V, Graziano S, et al. Immunohistochemical evaluation of E-cadherin and epidermal growth factor receptor in non-small cell lung cancer. Mod Pathol 1995;8:450-455.

14 Ramnath N, Hernandez FJ, Tan DF, et al. MCM2 is an independent predictor of survival in patients with non-small-cell lung cancer. J Clin Oncol 2001;19: 4259-4266.

15 Brambilla E, Travis WD, Colby TV, et al. The new World Health Organization classification of lung tumours. Eur Respir J 2001;18:1059-1068.

16 Mountain CF. Revisions in the International System for Staging Lung Cancer. Chest 1997;111:1710-1717.

17 Kononen J, Bubendorf L, Kallioniemi A, et al. Tissue microarrays for high-throughput molecular profiling of tumor specimens. Nat Med 1998;4:844-847.

18 Kaplan EL, Meier P. Nonparametric estimation from incomplete observation. J Am Stat Assoc 1958;53: 457-481.

19 Hoos A, Cordon-Cardo C. Tissue microarray profiling of cancer specimens and cell lines: opportunities and limitations. Lab Invest 2001;81:1331-1338.

20 Brundage MD, Davies D, Mackillop WJ. Prognostic factors in non-small cell lung cancer: a decade of progress. Chest 2002;122:1037-1057.

21 Kase S, Sugio K, Yamazaki K, et al. Expression of E-cadherin and beta-catenin in human non-small cell lung cancer and the clinical significance. Clin Cancer Res 2000;6:4789-4796.

22 Bremnes RM, Veve R, Gabrielson E, et al. Highthroughput tissue microarray analysis used to evaluate biology and prognostic significance of the E-cadherin pathway in non-small-cell lung cancer. J Clin Oncol 2002;20:2417-2428.

23 Ito K, Okamoto I, Araki N, et al. Calcium influx triggers the sequential proteolysis of extracellular and cytoplasmic domains of E-cadherin, leading to loss of betacatenin from cell-cell contacts. Oncogene 1999;18: 7080-7090.

24 Rawson RB. Regulated intramembrane proteolysis: from the endoplasmic reticulum to the nucleus. Essays Biochem 2002;38:155-168.

25 Han AC, Soler AP, Tang CK, et al. Nuclear localization of E-cadherin expression in Merkel cell carcinoma. Arch Pathol Lab Med 2000;124:1147-1151.

26 Herbst RS. ZD1839: targeting the epidermal growth factor receptor in cancer therapy. Expert Opin Investig Drugs 2002;11:837-849. 
27 Herbst RS, Langer CJ. Epidermal growth factor receptors as a target for cancer treatment: the emerging role of IMC-C225 in the treatment of lung and head and neck cancers. Semin Oncol 2002;29:27-36.

28 Veale D, Kerr N, Gibson GJ, et al. The relationship of quantitative epidermal growth factor receptor expression in non-small cell lung cancer to long term survival. Br J Cancer 1993;68:162-165.
29 Veale D, Ashcroft T, Marsh C, et al. Epidermal growth factor receptors in non-small cell lung cancer. $\mathrm{Br}$ Cancer 1987;55:513-516.

30 Inada S, Koto T, Futami K, et al. Evaluation of malignancy and the prognosis of esophageal cancer based on an immunohistochemical study (p53, E-cadherin, epidermal growth factor receptor). Surg Today 1999;29:493-503. 